\title{
Tratamiento del liquen plano oral: una revisión
}

\author{
García-Pola Vallejo MJ*, García Martín JM**
}

\section{RESUMEN}

El objetivo del presente estudio fue realizar una revisión sobre los actuales tratamientos del liquen plano oral (LPO). Antes de iniciar el tratamiento al paciente, debe de realizarse una biopsia para establecer el correcto diagnóstico. Dado que para el LPO no hay tratamiento curativo, el primer objetivo en los pacientes sintomáticos es el efecto paliativo. Entre las alternativas terapéuticas se incluyen: corticosteroides tópicos, sistémicos e intralesionales; retinoides tópicos o sistémicos; ciclosporina tópica, tacrolimus tópico, azatioprina, fototerapia y tratamiento quirúrgico.

Palabras clave: Liquen plano, tratamiento, corticosteroides, retinoides, ciclosporina, tacrolimus, azatioprina, fototerapia.

\section{SUMMARY}

The aim of the present study was to review about the current treatment of oral lichen planus (OLP). Before a patients is started on therapy a biopsy must be done to establish an accurate diagnosis. Because there is no curative therapy for OLP, the primary goal for symtomatic patients is palliative. Treatment modalities include the use of: topical, systemic and intralesional corticosteroids; topical and systemic retinoids, topical cyclosoporine; topical tacrolimus; azathioprine; phototherapy and surgical procedures.

Key words: Oral lichen planus, treatment, corticosteroids, retinoids, cyclosporine, tacrolimus, azathioprine, phototherapy.

Fecha de recepción: Diciembre 2007.

Aceptado para publicación: Diciembre 2007.

* Prof. Titular. Facultad de Medicina. Oviedo.

** Colaborador de Honor. Medicina Oral. Departamento de CIrugía y Especialidades Médico Quirúrgicas.

Facultad de Medicina. Oviedo.

García-Pola Vallejo, MJ, García Martín JM. Tratamiento del liquen plano oral: una revisión. Av. Odontoestomatol 2008; 24 (1): 45-53.

\section{INTRODUCCIÓN}

La indicación del tratamiento del liquen plano oral (LPO) se plantea una vez que se haya confirmado el diagnóstico de la enfermedad mediante la aplicación de criterios clínicos y estudios histopatológicos; y si fueran necesarios también inmunológicos $(1,2)$.

El enfoque terapéutico se realizará en función de los siguientes parámetros: la forma clínica; los síntomas; la localización y extensión del LPO; así como el fracaso de previos tratamientos (3).

Básicamente los objetivos del tratamiento del LPO serán: la eliminación de las lesiones atróficas y erosivas, controlar los síntomas y disminuir el potencial maligno de la lesión (4).

El primer paso a tener en cuenta es la eliminación de factores locales que pudieran exacerbar la le- 
sión para, a continuación, pautar el tratamiento específico del $L P O$.

\section{ELIMINACIÓN DE FACTORES QUE EXACERBAN LA LESIÓN}

\section{Hábitos orales}

Es importante minimizar el riesgo de desencadenar el fenómeno de Koebner, evitando hábitos como el mordisqueo labial, yugal o lingual (5). Además si es necesario, se pulirán las restauraciones desbordantes y la eliminación de las superficies anfractuosas dentarias, y se planteará la reparación o sustitución de las prótesis en mal estado (4).

\section{Control de la placa dental}

La placa dental pudiera ser un factor que indujera el fenómeno de Koebner (5). Holmstrup y cols, pusieron de manifiesto la mejoría de los pacientes con LP gingival cuando se les instruía en un programa de higiene oral mantenido durante un año (6).

Se ha documentado que en los pacientes con liquen plano de localización gingival, sometidos a tratamientos gíngivo-periodontales (tartrectomías y raspados gingivales) periódicos, una o dos veces al año, estos cuidados son beneficiosos para su evolución. También resulta de utilidad, realizar enjuagues con clorhexidina al 0,2 \% (sin alcohol) dos veces al día (7).

\section{Control del estrés}

Algunos pacientes han observado que su lesión empeora cuando están sometidos a estrés o ansiedad y que mejora al controlarlo (5).

\section{Dieta}

Debe aconsejarse a los pacientes mantener una dieta equilibrada y evitar la ingesta de alimentos que desencadenen dolor o exacerben las lesiones atrófico-erosivas (5).

\section{TRATAMIENTO DEL LPO}

El tratamiento del liquen plano podemos clasificarlo en tres niveles de actuación (3): I. Tratamientos de aplicación tópica, II. Tratamientos administrados por vía sistémica, y III. un tercer nivel: Miscelánea.

\section{TRATAMIENTO TÓPICO}

\section{CORTICOSTEROIDES TÓPICOS}

\section{Principio activo}

Los corticosteroides (CT) de aplicación tópica se clasifican en siete grupos (tabla I), en función de su potencia de acción, ya que un mismo principio activo al que se le modifica su concentración, se le está variando su potencia de acción (8). Con frecuencia en la literatura relacionada con la efectividad de los CT, para simplificar esta clasificación, se reduce a cuatro grupos (9): Potencia de acción baja (ej.: hidrocortisona 1\%), media (ej.: acetónido de triamcinolona al $0,1 \%$ ), potente (ej.: dipropionato de betametasona al $0,05 \%$ ) y muy potente (ej.: propionato de clobetasol al 0,05\%).

\section{Vehículo o base}

El vehículo puede modificar la eficacia del CT, y ello representa una de las limitaciones en su aplicación sobre la mucosa oral. Sobre la piel se adhieren mejor utilizando pomadas o ungüentos, pero sobre la mucosa húmeda la mejor estabilidad de los CT se consigue mediante geles y soluciones. Entre ellos los más utilizados son el gel de Orabase y la solución acuosa (10). El Orabase es una pasta adhesiva formada por carboximetil celulosa sódica $(16,7 \%)$, pectina $(16,7 \%)$, gelatina $(16,7 \%)$, polieno $(2,5 \%)$, y parafina líquida $(47,4 \%)$.

Hegarty et al (11) en un estudio comparativo de formas farmacéuticas, destacaron la predilección de los pacientes por la aplicación del CT en formato spray frente a los enjuagues.

Otros vehículos y formas farmacéuticas utilizados son: pastas dentífricas sin componentes activos (10), 


\begin{tabular}{|l|l|}
\hline \multicolumn{2}{|c|}{$\begin{array}{c}\text { TABLA 1.- CLASIFICACIÓN DE LOS } \\
\text { CORTICOSTEROIDES (CT) SEGÚN SU } \\
\text { POTENCIA DE ACTUACIÓN. }\end{array}$} \\
$\begin{array}{l}\text { LA MENOR POTENCIA LE CORRESPONDE } \\
\text { A LA UNIDAD DE HIDROCORTISONA } \\
\text { (GRUPO 7) (8) }\end{array}$ \\
\hline $\begin{array}{l}\text { Clase de CT } \\
\text { según } \\
\text { su potencia }\end{array}$ & \multicolumn{1}{c|}{ Nombre del fármaco } \\
\hline Clase 1 & Propionato de clobetasol 0,05\%. \\
\hline Clase 2 & $\begin{array}{l}\text { Dipropionato de betametasona } \\
0,05 \% .\end{array}$ \\
\hline Clase 3 & Valerato de betametasona $0,1 \%$. \\
\hline Clase 4 & Acetónido de fluocinolona $0,2 \%$ \\
\hline Clase 5 & $\begin{array}{l}\text { Acetónido de triamcinolona al } \\
0,1 \% .\end{array}$ \\
\hline Clase 6 & Acetónido de fluocinolona $0,01 \%$ \\
\hline Clase 7 & Hidrocortisona $1 \%$ \\
\hline
\end{tabular}

propilenglicol (asociado a etanol y sorbitol) (12), así como aquellos que se obtienen de variaciones galénicas a expensas de lípidos microesféricos (13).

\section{Forma de aplicación}

Los pacientes deben ser orientados respecto a la aplicación del preparado. El contacto prolongado del CT proporciona un incremento de la eficacia. Cuando la base es un gel, el paciente debe aplicar el CT con la cánula (si se dispensa con el producto) o con una torunda de algodón o bastoncillo de los oídos, y mantenerlo contra la mucosa durante al menos un minuto. El paciente no debe comer o beber en lo 3060 minutos posteriores a la aplicación.

Cuando la afectación es gingival y/o en paladar se pueden elaborar unas cubetas con soporte dentario y cuyo diseño vendrá determinado por la extensión de la lesión (14). La aplicación de las cubetas es de 5 minutos (14) a 30 minutos (15).
Si el CT es pautado en solución, los enjuagues se realizan manteniendo el contenido durante minutos en la boca. Es necesario advertir al paciente que no trague la solución y que no coma ni beba inmediatamente, tras realizar los enjuagues.

\section{Posología}

El número de aplicaciones depende de la potencia del CT. Por tanto, aquellos CT de potencia baja o media se pautarán de 5 a 6 veces al día, mientras que los de potencia alta o muy alta se pautan 2 ó 3 veces al día. Una vez que la evolución de la sintomatología y la forma clínica sea favorable, la posología debe ser disminuida de forma progresiva. La duración del tratamiento oscila considerablemente de unos pacientes a otros.

\section{Efectos adversos}

Como comentamos previamente, los pacientes no deben tragar el medicamento residual, para evitar al máximo los efectos adversos. (15).

Cuando se utilizan los CT en forma de enjuagues la absorción sistémica es más elevada y por tanto los efectos adversos son mayores $(16,17)$. No se han descrito complicaciones suprarrenales $(18,19)$, siendo el efecto secundario más frecuente la candidiasis (20-22), seguido de otros como hirsutismo, petequias y "facies de luna llena" (17). Para evitar la candidiasis post-tratamiento, está justificada la indicación de soluciones de nistatina (23).

\section{CiclosPoRINA TÓPICA}

La ciclosporina es un fármaco inmunosupresor que se utiliza en aquellos pacientes con liquen plano gingival y/ o con afectación difusa que no responde al tratamiento con CT (3).

Se aconseja la utilización de aceite de oliva de acidez $0,4^{\circ}$ como vehículo (24). Los pacientes deben distribuir por toda la mucosa oral, 3-5 ml de la ciclosporina durante 5 minutos, 3 veces al día. No deben comer ni beber durante los 30 minutos in- 
mediatos después del tratamiento (3). En las formas clínicas atróficas y erosivas, después de 6-8 semanas de tratamiento, suele obtenerse un resultado favorable

La administración de la ciclosporina de forma tópica presenta una absorción sistémica baja, por lo que su efectividad no es correlativa con los niveles en sangre (25). Entre los efectos adversos que desencadena su aplicación son el mal sabor y la sensación de ardor.

El coste del tratamiento con ciclosporina es elevado, por lo que se ha intentado conseguir una dosis terapéutica con menor concentración de principio activo. La dosis de $500 \mathrm{mg}$ (5 ml), obtuvo efectos beneficiosos frente a la utilización de un placebo (26).

Recientes estudios han puesto en evidencia la menor efectividad de las ciclosporinas, si se compara con la triamcinolona (27), y el clobetasol (28) de aplicación tópica para el tratamiento del LPO.

\section{TACROLIMUS TÓPICO}

El tacrolimus es un macrólido que inhibe la activación de las células $T$, a una concentración de 10 a 100 veces más baja que la ciclosporina. El tacrolimus se puede dispensar a una concentración del $0,1 \%$ en Orabase, una o dos veces al día (29), y del $1 \%$ (30). Para algunos patólogos orales los efectos adversos son mínimos (31), y no se ha registrado su absorción sistémica (29), sin embargo se ha descrito un caso de malignización lingual tras la aplicación de tacrolimus (32)

La respuesta al tratamiento es rápida pero la mayoría de los pacientes necesitan prolongar el tratamiento y pautar un tratamiento de mantenimiento para evitar las recurrencias $(33,34)$.

\section{RETINOIDE TÓPICO}

La aplicación tópica de retinoides ofrece una respuesta completa o parcial de la lesión entre el 71 y $94 \%$ de los pacientes, pero tras suspender el tratamiento las recidivas son frecuentes (3).
La eficacia es observada a partir de concentraciones del 0,1\% del ácido retinoico (35) o retinaldehido (36), no se observa mejoría en proporciones del 0,05\% (37). En un estudio comparativo de la eficacia entre el ácido retinoico al 0,05\% y la fluocinolona al 0,1\%, ambos aplicados tópicamente en orabase, se llegó a la conclusión de que era más eficaz el CT que el retinoide sobre los LPO erosivos (37).

Como efectos adversos se han descrito el enrojecimiento de la mucosa, y sensación de quemazón (38).

\section{APLICACIÓN INTRALESIONAL}

La aplicación del CT intralesional, se realiza directamente sobre las zonas erosivas del LPO, cuando éstas se localizan sobre mucosa no adherida, fundamentalmente en las mucosas yugales o linguales. Su aplicación puede ir precedida o no de la inyección de un anestésico, para aliviar la molestia del infiltrado.

El CT utilizado es el acetónido de triamcinolona a dosis de 10-20-40 mg/ml. Dado que su acción es "retardada", su aplicación sólo se puede repetir semanalmente (39), durante 2 ó 4 semanas (4), o mensualmente (3). No suele desencadenar efectos adversos (39).

\section{TRATAMIENTOS SISTÉMICOS}

\section{CORTICOSTEROIDES SISTÉMICOS}

Una alternativa propuesta en situaciones de exacerbación clínica del LPO, es la pauta consistente en una baja dosis de CT por vía oral durante un periodo de tiempo corto, hasta que sean efectivos los CT tópicos (5). Se utilizan dosis entre los 30-60 mg/día, una vez al día, por la mañana, durante 1 ó 3 semanas, manteniéndolo a días alternos (40). Debe recomendarse la disminución progresiva de la dosis seleccionada para evitar los efectos adversos más frecuentes como son: molestias gastrointestinales, cambios conductuales, cataratas, cara de luna, poliuria, insomnio, candidiasis, hiperglucemia, etc (41).

Algunos trabajos han puesto de manifiesto que la asociación de CT sistémicos más la aplicación tópi- 
ca, no actúan de forma más eficaz que cuando éstos se pautan solos. $(20,42,43)$.

En otros trabajos de investigación se constató el beneficio a largo plazo empleando pautas de asociación de CT por vía oral (a bajas dosis) con inmunomoduladores. La combinación y dosis recomendadas son: levamisol a razón de $50 \mathrm{mg}, 3$ veces al día y prednisolona $5 \mathrm{mg}, 3$ veces al día, durante 3 días seguidos a la semana $(44,45)$.

\section{RETINOIDES SISTÉMICOS}

Diferentes estudios han puesto de manifiesto que la utilización de isotretionina a dosis de 10-60 mg/día produce escasos efectos beneficiosos sobre el liquen plano erosivo $(45,46)$.

Dosis más elevadas de etretinato, 75 mg/día, durante dos meses, proporcionan una mejoría clínica de las lesiones (47), pero a largo plazo (un año), los pacientes están en la misma situación que antes de iniciar el tratamiento (48). Con estas dosis además se padecen muchos efectos adversos e incluso algunos pacientes, tienen que suspender el tratamiento $(47)$.

Otra pauta propuesta es la administración de 0,6 $\mathrm{mg} / \mathrm{kg} /$ día, seguido de un mantenimiento con 0,3 $\mathrm{mg} / \mathrm{kg} /$ día o bien la aplicación tópica de etretionina al 0,1\% en una base adhesiva dos veces al día (49). A pesar de estas consideraciones, una vez que se suspende el tratamiento el LPO recurre (50).

\section{MISCELÁNEA}

\section{Azatioprina}

La azatioprina es empleada para el tratamiento del liquen plano por su efecto inmunosupresor. Las dosis recomendadas son 50-100 mg/día. Puede ser aplicado en combinación con la terapia de CT para reducir la posología de éstos (40).

Los efectos adversos son muy numerosos y de diversa índole, motivo por el cual se debe extremar su pauta: Los más importantes son: náuseas, vómitos, diarrea, pancreatitis, supresión de médula ósea, hepatotoxicidad y retinopatías. Se debería utilizar con precaución en pacientes con disfunción hepática y renal, así como con problemas hematológi$\cos (50)$.

La utilización con éxito de la Azatioprina de forma tópica, en gel y solución con una base de metilcelulosa, para el tratamiento de las lesiones erosivas en pacientes sometidos a trasplantes (51), abre una puerta para su aplicación en pacientes con liquen plano.

\section{Fototerapia}

Se ha utilizado para el tratamiento del LPO la aplicación de radiaciones ultravioleta de onda larga (UVA), de forma única o precedida de la administración oral o tópica de psoralenos (PUVA). El número de aplicaciones suele ser de hasta 20 (52) con intervalos de 2 a 3 días $(53,54)$. La administración del 8 metoxypsoraleno es a razón de $0,6 \mathrm{mg} / \mathrm{kg}$, dos horas antes de la sesión de UVA.

Los efectos adversos que desencadenan los psoralenos son frecuentes, y entre ellos destacan: las náuseas, vértigo, y parálisis.

El riesgo de padecer cáncer de mucosa oral tras la aplicación de PUVA podría ser menor que cuando se aplica sobre la piel, al ofrecer ésta menor resistencia a la acción de los psoralenos.

La fotoquimioterapia extracorporal (fotoféresis) (55), es otra alternativa compleja para el tratamiento de liquen plano refractario a otras terapias. Mediante esta técnica se separan células mononucleares de la sangre del paciente, se mezclan con psoraleno y se irradian con luz ultravioleta (54).

\section{Tratamiento quirúrgico}

Entre los tratamientos quirúrgicos se incluyen la escisión, la criocirugía, la utilización de láser (56), e injertos de mucosa palatina sobre mucosa gingival $(57,58)$. 
El láser de $\mathrm{CO}_{2}$ está indicado en el liquen plano en placa (56), ya que en otras formas clínicas de LPO las recurrencias son más frecuentes $(3,59)$. La utilización del láser $\mathrm{CO}_{2}$ presenta unas ventajas con respecto a la cirugía convencional: eliminación de lesiones extensas, visibilidad del campo operatorio sin hemorragia, postoperatorio con escasas complicaciones y reparación ad integrum (56).

\section{BIBLIOGRAFÍA}

1. WHO Collaborating Centre for Oral Precancerous Lesions. Definition of leukoplakia and related lesions: an aid to studies oral precancer. Oral Surg Oral Med Oral Pathol 1978;46:51839.

2. Firth NA, Rich AM, Radden RG, Reade PC. Assessment of the value of immunofluorescence microscopy in the diagnosis of oral mucosal lichen planus. J Oral Pathol Med 1990; 19:295-7.

3. Eisen D. Evaluating and treating patients with oral lichen planus. Dermathologyc Therapy 2002; 15: 206-17.

4. Esisen D, Carrozo M, Bagán Sebastian JV, Thongprasson K. Oral lichen planus: clinical features and management. Oral Dis 2005; 11:338-49.

5. Eisen D. The Therapy of Oral Lichen Planus. Clin Rev Oral Biol 1993; 4: 141-58.

6. Holmstrup P, Schiotz A, Westergaard J. Effect of dental plaque control on gingival lichen planus. Oral Surg Oral Med Oral Pathol 1990; 69: 585-90.

7. Guiglia R, Liberto CD, Pizzo G, et al. A combined treatment regimen for desquamative gingivitis in patients with oral lichen planus. J Oral Pathol 2007; 36:110-6.

8. Stoughton R, Cornell R. Corticosteroids. En: Fitzpatrick Th. Dermatology in general medicine. McGraw-Hill, Inc, 1993: 2846-50.
9. Giménez Arnau AM. Corticosteroides tópicos (II): tipos, indicaciones, efectos adversos. Acta Dermatolog 1997; 819: 573-82.

10. González-Moles MA, Scully C. Vesiculo-erosive Oral Mucosal Disease-Management with Topical Corticosteroids: (1) Fundamental Principles and Specific Agents Available. J Dent Res 2005; 84(4): 294-301.

11. Hegarty AM, Hodgon TA, Lewsey JD, Porter SR. Fluticasone propionate spray and betamethasone sodium phosphate mouthrinse: A randomized crossover study for the treatment of symptomatic oral lichen planus. J Am Acad Dermatol 2002; 47: 271-9.

12. Ungphaiboon S, Nittayananta W, Vuddhakull W, Maneenuan D, Kietthubithew S, Wongpoowarak W, Phadoongsombat $N$. Formulation and efficacy of triamcinolone acetonide mouthwash for treating oral lichen planus. Am J Health Syst Pharm 2005;62:485-91.

13. Campisi G, Giandalia G, de Caro V, di Liberto C, Arico P, Giannola L. A new delivery system of clobetasol-17-propionate (lipid-loaded microspheres $0.025 \%$ ) compared with a conventional formulation in a hydrophilic phase $0.025 \%$ ) in topical treatment of atrophic/erosive oral lichen planus. A Phase IV, randomized, observer-blinded, parallel group clinical trial. Br J Dermatol 2004; 150: 984-90.

14. González-Moles MA, Ruiz-Avila I, Rodriguez-Archilla A, Morales-García P, Mesa-Aguado F, BasconesMartínez A. Treatment of severe erosive gingival lesions by topical application of clobetasol propionate in custom trays. Oral Surg Oral Med Oral Pathol Oral Radiol Endod 2003; 95: 688-92.

15. Huber MA. Oral lichen planus. Quintessence 2004;35:731-52.

16. González-Moles MA, Morales P, Rodríguez-Archilla A, Ruiz-Avila I, Gonzalez-Moles S. Treatment of severe chronic erosive lesions with clobetasol propionate in aqueous solution. Oral Surg Oral Med Oral Pathol Oral Radiol Endod 2002·, 93: 264-70. 
17. González-Moles MA, Scully C. Vesiculo-erosive Oral Mucosal Disease-Management with Topical Corticosteroids: (2) Protocols, Monitoring of Effects and Adverse Reactions, and the Future Principles and Specific Agents Available. J Dent Res 2005; 84(4): 302-8.

18. Thongprasson $\mathrm{K}$, Luengvisut $\mathrm{P}$, Wongwatamakj $\mathrm{A}$, Boonjatturus $\mathrm{C}$. Clinical evaluation in treatment of oral lichen planus with topical fluocinolone acetonide: a 2-year follow-up. J Oral Pathol 2003; 32: 315-22.

19. Carbone M, Goss E, Carrozo M, Castellano S, Conroto D, Broccoletti R, Gondolfo SJ. Systemic and topical corticosteroid treatment of oral lichen planus: a comparative study with long-term follow-up. J Oral Pathol 2003;32:323-9.

20. Vincent SD., Fotos PG, Baker KA; Williams TP. Oral lichen planus: The clinical, historical and therapeutic features of 100 cases. Oral Surg Oral Med Oral Pathol 1990;70:165-71.

21. Aguirre JM, Bagán JV, Rodríguez C, Jiménez Y, Martínez-Conde R, Diz de Rojas F, Ponte A. Efficacy of mometasone furoate microemulsion in the treatment of erosive-ulcerative oral lichen planus: pilot study. J Oral Pathol 2004; 33:381-5.

22. González-García A, Diniz-Freitas M, Gándara-Vila P, Blanco-Carrión A, García-García A, GándaraRey JM. Triamcinolone acetonide mouth rinses for treatment erosive oral lichen planus: efficacy and risk of fungal over-infection. Oral Dis 2006; 12:559-65.

23. Cerero R, García-Pola MJ. Tratamiento del liquen plano oral. Med Oral 2004;9:124.

24. Lopez Lopez J, Rosello Llabres X. Ciclosporine A, an alternative to the oral lichen planus erosive. Bull Group Int Rech Sci Stomatol Odontol 1995; 38:33-8.

25. Eisen D, Ellis C. Topical cysclosporine for oral mucosal disorders. J Am Acad Dermatol 1990; 23: 1259-64.
26. Harpenau LA, Plenons JM, Rees TD. Effectiveness of a low dose of cyclosporine in the management of patients with oral erosive lichen planus. Oral Surg Oral Med Oral Pathol Oral Radiol Endod 1995;80(2):161-7.

27. Yoke PC, Tin GB, KimM, et al. A randomized controlled trial to compare steroid with cyclosporine for the topical treatment of oral lichen planus. Oral Surg Oral Med Oral Pathol Oral Radiol Endod 2006;102:47-55.

28. Conrotto D, Carbone M, Carrozo M, Arduino P, Broccoletti R, Pentenero M, Gandolfo S. Ciclosporine vs. Clobetasol in the topical management of atrophic and erosive oral lichen planus: a double blind, randomized controlled trial. Br J Dermatol 2006;154:139-45.

29. Thomson MA, Hamburger J, Stewart DG, Lewis $\mathrm{HM}$. Treatment of erosive oral lichen planus with topical tacrolimus. J Dermatol Treat 2004; 15: 308-14.

30. Esquivell-Pedraza I, Fernández-Cuevas L, OrtizPedrza G, Reyes-Gutiérrez E, Orozco-Topete R. Treatment of oral lichen planus with topical pimecrolimus 1\% cream. Br J Dermatol 2004; 150:771-3.

31. Lozada-Nur F, Sroussi H. Tacrolimus powder in Orabase $0.1 \%$ for the treatment of oral lichen planus and oral lichenoid lesions: An open clinical trial. Oral Surg Oral Med Oral Pathol Oral Radiol Endod 2006;102:744-9.

32. Becker JC, Houben R, Vetter CS, Broecker EB. The carcinogenic potential of tacrolimus ointment beyond immune suppression: a hypothesis creating case report. BMC Cancer 2006;6:7.

33. Byrd J, Davis M, Bruce A, Drage L, Rogers R. Response of Oral Lichen Planus to Topical Tacrolimus in 37 patients. Arch Dermatol 2004; 140:1508-12.

34. Schichinohe R, Shibaki A, Nishie W, Tateischi Y, Shinizu H. Successful treatment of severe 
recalcitrant erosive oral lichen planus with topical tacrolimus. J Eur Acad Dermatol Venerol 2006; 20: 66-8.

35. Regezi JA, Ellis C, Stewart J, Giustina T. Histologic changes associated with the topical use of isotretinoin on oral lichen planus. Oral Surg Oral Med Oral Pathol 1985; 61 (5): 479-84.

36. Boisnic S, Licu D Ben L, Branchet-Gumila MC, Szpirglas H, Duouy P. Topical retinaldehyde treatment in oral lichen planus and leukoplakia. Int J Dermatol React 2002; 24(4): 123-30.

37. Buajeeb W, Kraivaphan P, Pobrurksa C. Efficay of topical retinoic acid compared with topical fluocinolone acetonide in the treatment of oral lichen planus. Oral Surg Oral Med Oral Pathol 1997; 83(1): 21-5.

38. Giustina TA, Stewart JC, Ellis CN, Regezi JA, Annesley T, Woo TY, Voohees JJ. Topical application of isotretinoin gel improves oral lichen planus. A double-blind study. Arch Dermatol 1986; 22(5):534-6.

39. Xia J, LiC, Hong Y, Yang L, Huang Y, Cheng B. Short-term clinical evaluation of intralesional triamcinolone acetonide injection for ulcerative oral lichen planus. J Oral Pathol Med 2006; 35: 327-31.

40. Lozada-Nur F, Miranada C. Oral lichen planus: topical and systemeic therapy. Semin Cuta Med Surg1997;4:295-300.

41. Schimmer B, Parker K. Hormona supranorrenocorticotrópica: esteroides suprarrecorticales y sus análogos sintéticos; inhibidores de la síntesis y los efectos de las hormonas suprarrenocorticales. En: Pasman JG, Las bases farmacológicas de la terapéutica. México, $8^{a}$ ed: McGraw-Hill Interamericana Editores:1996:1551-9.

42. Silverman SJr; Gorsky M, Lozada-Nur F, Giannoti K. A prospective study of findings and management in 214 patients with oral lichen planus. Oral Surg Oral Med Oral Pathol 1991; 72:665-70.
43. Lu SY, Chen W, Eng H. Dramatic response to levamisole and low-dose prednisolone in 23 patients with oral lichen planus. Oral Surg Oral Med Oral Pathol Oral Radiol Oral Endod 1995; 80: 705-9.

44. Lu SY, Chen W, Eng H. Response to levamisole and low-dose prednisolone in 41 patients with chronic oral ulcers. Oral Surg Oral Med Oral Pathol Oral Radiol Endod 1998;86:438-45.

45. Camisa C, Allen CM. Treatment oral erosive lichen planus with systemic isotretinoin. Oral Surg Oral Med Oral Pathol 1986;62(4):393-6.

46. Woo TY. Systemic isotretinoin treatment of oral and cutaneous lichen planus. Cutis 1985;35: 385-6.

47. Hersle K, Mobacken H, Sloberg K, Thilander H. Severe oral lichen planus : treatment with an aromatic retinoid (etretinate). Br J Dermatol 1982; 106(1):77-80.

48. Gorsky M, Ravoiv M. Efficacy of etretinate (Tigason) in symptomatic oral lichen planus. Oral Surg Oral Med Oral Pathol 1992;73(1):52-5.

49. Tradati N, Chiesa F, Rossi N, Grigolato R, Fornelli F, Costa A, de Palo G. Successful topical treatment of oral lichen planus and leukoplakias with fenretinide (4-HPR). Cancer Lett 1994;30; 76(2-3):109-11.

50. Epstein JB, Gorsky M, Epstein M, Nantel S. Topical azathioprine in the treatment of immunemediated chronic oral inflammatory conditions. Oral Surg Oral Med Oral Pathol Oral Radiol Endod 2001;91:56-61.

51. Seoane J, Vazquez J, Romero MA, Aguado A, Pomareda M. Photochemotherapy in the treatment of oral erosive liche planus. Acta Otorrinolaringol Esp 1997;48(3):251.3.

52. Lundquist G, Forsgren H, Gajecki M, Emtestam L. Photochemotherapy of oral lichen planus. Oral Surg Oral Med Oral Pathol Radiol Endod 1995;79: 554-8. 
53. Guyot A, Farhi D, Ingen-Housz-Oro S, Bussel A, Parquet $\mathrm{N}$, Rabian $\mathrm{C}$, Bachelez $\mathrm{H}$, Francès $\mathrm{C}$. Treatment of refractary erosive lichen planus with extracorporeal photochemoteray: 12 cases. $\mathrm{Br} \mathrm{J}$ Dermatol 2007;156:553-6.

54. Beetherel PA, Bussel A, Chosidow O, Rabian C, Piette JC, Francès C. Extracorporeal photochemotherapy for chronic erosive lichen planus. Lancet 1998;351:805.

55. Loh HS. A clinical investigation of the management of oral lichen planus with $\mathrm{CO}_{2}$ laser surgery. J Clin Laser Med Surg 1992;10(6):445-9.

56. Huerta Leteurtre N, Bagán Sebastián JV, Cardona Tortajada F, Lloria de Miguel E, Jiménez Soriano Y, Basterra Alegría J. Liquen plano oral en placas y leucoplasia oral homogénea: resultados comparativos tras el tratamiento de estas lesiones con láser $\mathrm{CO}_{2}$. Acta Otorrinolaringol Esp 1999;50(7):543-7.
57. Tamizi M, Moayedi $M$. Treatment of gingival lichen planus with a free gingival graft: a case report. Quintessence Int 1992; 23(4):249-51.

58. Áxell T, Henriksen BM. Treatment of gingival lichen with free palatal grafts. J Oral Pathol 2007; 36:105-9.

59. Kollner K, Winnershoff $M$, Landhaler $M$, Hohenleutner $U$. Treatment of oral lichen planus with 308 UVB excimer laser-early preliminary results in eight patients. Lasers Surg Med 2003; 33(3):158-60.

\section{CORRESPONDENCIA}

María José García-Pola Vallejo

Facultad de Medicina

Catedrático José Serrano, s/n

33006 Oviedo

Correo electrónico: mgpola@fade.es 